\title{
INVARIANT MEANS ON SPACES OF CONTINUOUS OR MEASURABLE FUNCTIONS $\left({ }^{1}\right)$
}

\author{
BY \\ CHIVUKULA RAMAMOHANA RAO ${ }^{2}$ )
}

1. Introduction. Invariant means on spaces of functions have been studied by von Neumann [7], Banach [2], Day [4], [5] and others. Day's Amenable semigroups [5] presents a comprehensive summary of the earlier work and many new results. Let $\Sigma$ be an abstract group or semigroup and $m(\Sigma)$ the Banach space of all bounded real-valued functions on $\Sigma$ with the supremum norm. A mean on $m(\Sigma)$ is a positive linear functional of norm one. Left and right translation operators, respectively denoted by $l_{\sigma}$ and $r_{\sigma}$, are defined as: $\left(l_{\sigma} x\right)\left(\sigma^{\prime}\right)=x\left(\sigma \sigma^{\prime}\right)$ and $\left(r_{\sigma} x\right)\left(\sigma^{\prime}\right)=x\left(\sigma^{\prime} \sigma\right)$ for $x$ $\in m(\Sigma)$ and $\sigma, \sigma^{\prime} \in \Sigma$. Let, as usual, $\mathscr{L}(X)$ denote the algebra of bounded linear operators on a Banach space $X$ into itself. Clearly $l_{\sigma}$ and $r_{\sigma} \in \mathscr{L}(m(\Sigma))$. A mean $\mu$ is called (left) [ right] invariant if $\left(l_{\sigma}^{*} \mu=\mu\right)\left[r_{\sigma}^{*} \mu=\mu\right]$ for all $\sigma$ in $\Sigma$. Day calls $\Sigma$ (left) [ right] amenable if there exists a (left) [ right] invariant mean on $m(\Sigma)$. Various results on amenability of groups and semigroups are listed in $\$ 4$ of Day [5].

In the work mentioned above $\Sigma$ is an abstract group or semigroup. As such it can be considered as a discrete topological space and then $m(\Sigma)$ $=C(\Sigma)$, the space of all bounded continuous real-valued functions. In the case of a group, we may consider $\Sigma$ as a measure space with discrete Haar measure and then $m(\Sigma)=M B(\Sigma)$, the space of all bounded measurable functions. However, if the topology of $\Sigma$ is not discrete then $M B(\Sigma)$ and $C(\Sigma)$ are different from $m(\Sigma)$. The purpose of this paper is to study this situation. Henceforth $\Sigma$ will stand for a locally compact topological group, unless otherwise explicitly stated. From the theory of measure, it is well know that $\Sigma$ can be equipped with a left or right invariant Haar measure. It may be noted that neither the concept of measurability nor the concept of a set of measure zero are dependent on the particular measure introduced. We shall now list the various spaces that will enter our discussion and give some definitions and preliminary results.

\footnotetext{
Received by the editors July 30, 1962 and in revised form, August 28, 1963.

( $\left.{ }^{1}\right)$ This paper is based upon a dissertation written under the direction of Professor Mahlon $\mathrm{M}$. Day and submitted in partial fulfillment of the requirements for the $\mathrm{Ph}$. $\mathrm{D}$. degree at the University of Illinois (Urbana).

$\left({ }^{2}\right)$ Author partly supported by the National Science Foundation under contract No. NSF G 12171.
} 
$m(\Sigma)$ : The space of all bounded real-valued functions $x$ on $\Sigma$ with $\|x\|$ $=\sup _{\sigma \in \Sigma}|x(\sigma)|$.

$C(\Sigma)$ : The subspace of $m(\Sigma)$ consisting of continuous functions.

$l_{1}(\Sigma)$ : The space of all real-valued functions $\theta$ on $\Sigma$ with

$$
\|\theta\|=\sum_{\sigma \in \Sigma}|\theta(\sigma)|<+\infty .
$$

$M B(\Sigma)$ : The space of all real-valued measurable, essentially bounded (with respect to Haar measure) functions $x$ on $\Sigma$ with

$$
\|x\|=\underset{\sigma \in \Sigma}{\operatorname{ess} \sup }|x(\sigma)| \text {. }
$$

Two functions differing only on a set of measure zero are considered as the same.

$L_{1}(\Sigma)$ : The space of all real-valued $h$-summable $\left({ }^{3}\right)$ functions $\theta$ on $\Sigma$ with $\|\theta\|=\int_{\Sigma}|\theta(\sigma)| d h(\sigma)$.

All these spaces are Banach spaces. Besides these, we consider certain subspaces of $C(\Sigma)$ and $M B(\Sigma)$. They will be defined in the proper context. Let $X$ stand for a subspace of either $m(\Sigma)$ or $M B(\Sigma)$, containing the constant functions. It is well known that $m(\Sigma)=l_{1}(\Sigma)^{*}$ and $M B(\Sigma)=L_{1}(\Sigma)$. On subspaces $X$ we sometimes use the $w^{*}$-topology (weak topology in the sense of Bourbaki [3]).

Definition 1. A mean $\mu$ on $X$ is an element of $X^{*}$ such that: (a) if $e$ is the constantly one function on $\Sigma$ then $\mu(e)=1$; and (b) if $x(\sigma) \geqq 0$ for every $\sigma \in \Sigma$ then $\mu(x) \geqq 0$.

(A) Each mean $\mu$ has the property: (c) $\|\mu\|=1$.

(B) If $\mu \in X^{*}$ satisfies any two of the three conditions (a), (b) and (c) it satisfies the third and so is a mean.

(C) The set of means on $X$ is nonempty, convex and $w^{*}$-compact.

These three statements are proved in the same way as (A), (B) and (C) on p. 573 in [5].

Definition 2. (i) $\theta \in l_{1}(\Sigma)$ is called a countable mean if $\theta(\sigma) \geqq 0$ for all $\sigma$ and $\sum_{\sigma \in \Sigma} \theta(\sigma)=1$; it is called a finite mean if, in addition, the set $\{\sigma \mid \theta(\sigma)>0\}$ is finite. (ii) $\theta \in L_{1}(\Sigma)$ is called a compact mean if $\theta \geqq 0$ for almost all $\sigma$ and the set $\{\sigma \mid \theta(\sigma)>0\}$ is compact up to a set of measure zero and $\|\theta\|=1$.

Note. If $\Sigma$ is discrete then a compact mean becomes a finite mean.

Let $Q$ and $q$ be respectively the imbeddings of $L_{1}(\Sigma)$ and $l_{1}(\Sigma)$ into $M B(\Sigma)^{*}$ and $m(\Sigma)^{*}$; let $J$ and $j$ be the injection maps of $X$ into $M B(\Sigma)$ or $m(\Sigma)$. It is easy to see that $J^{*}$ and $j^{*}$ respectively carry the set of means

$\left({ }^{3}\right) h$ stands for a Haar measure. $L_{1}(\Sigma)$ is independent of the choice of the measure but the norm is not. 
on $M B$ and $M$ onto the set of means on $X$.

$\left(D_{1}\right)$ If $\Phi$ is the set of finite means then $q \Phi$ is $w^{*}$-dense in the set of means on $m(\Sigma)$.

$\left(D_{2}\right)$ If $\Psi$ is the set of compact means then $Q \Psi$ is $w^{*}$-dense in the set of means on $M B(\Sigma)$.

$\left(\mathrm{D}_{3}\right)$ If $X \subseteq m(\Sigma)$ then $j^{*} q \Phi$ is $w^{*}$-dense in the set of means on $X$.

$\left(\mathrm{D}_{4}\right)$ If $X \subseteq M B(\Sigma)$ then $J^{*} Q \Psi$ is $w^{*}$-dense in the set of means on $X$.

These four propositions are easy consequences of the general theory of Banach spaces.

We have already defined the left and right translation operators $l_{\sigma}$ and $r_{\sigma}$. A subspace $X$ is called left invariant if with each element $x$, it contains the set $\left\{l_{\sigma} x \mid \sigma \in \Sigma\right\}$. Right invariance is defined analogously and $X$ is called invariant if it is both. The operators $r_{\sigma}$ and $l_{\sigma}$ are meaningful on any invariant $X$ and we use the same symbols whether they are considered to be in $\mathscr{L}(m(\Sigma))$ or $\mathscr{L}(M B(\Sigma))$ or, more generally, in $\mathscr{L}(X)$. It is easy to verify that the correspondence of $\sigma$ with $r_{\sigma}$ is a homomorphism of $\Sigma$ into $\mathscr{L}(X)$, and that of $\sigma$ with $l_{\sigma}$ is an anti-homomorphism. Also $\left\|l_{\sigma}\right\|=\left\|r_{\sigma}\right\|=1$. These maps are generally not isomorphic but we shall show, in the next section, that under certain conditions they are not only isomorphisms but also homeomorphisms of $\Sigma$ into $\mathscr{L}(X)$ in the weak operator topology.

Definition 3. $\mu \in X^{*}$ is called (right) [ left] invariant if $\left(\mu\left(r_{\sigma} x\right)=\mu(x)\right)$ $\left[\mu\left(l_{\sigma} x\right)=\mu(x)\right]$ for all $x \in X$ and $\sigma \in \Sigma$.

Definition 4. $A$ (right) [ left] invariant $X$ is called (right) [left] amenable if there exists a (right) [ left] invariant mean on it.

As was mentioned earlier, most of the work on invariant means has been in the case of a discrete $\Sigma$ and the space $m(\Sigma)$ associated with it $\left({ }^{4}\right)$. This space enjoys a very nice property called introversion (Day's terminology) and almost all known results make use of it. It will be seen from the discussion in the next section that introversion is not a property easy to come by. For example, the space of bounded continuous functions on the real line is not introverted. $\$ 2$ is devoted to a discussion of introversion and some of its consequences. We show that there exist unique maximal left, right and (two-sided) introverted subspaces in any closed invariant $X$. $\$ 3$ contains some results on amenability.

2. Introversion. Let $X$ be an invariant subspace and $x \in X$ and $\xi \in X^{*}$. Define $\left(\xi_{l} x\right)(\sigma)=\xi\left(l_{\sigma} x\right)$ and $\left(\xi_{r} x\right)(\sigma)=\xi\left(r_{\sigma} x\right)$ for all $\sigma$ in $\Sigma$. It is obvious that $\xi_{l} x$ and $\xi_{r} x \in m(\Sigma)$ no matter what $X$ is.

$\left({ }^{4}\right)$ Rosen [8] considers topological semigroups and the space $C(\Sigma)$. But very soon he imposes on them the condition WCR (weakly continuously representable) which, in the present terminology, means that $C(\Sigma)$ is introverted. 
Definition 5. Say that a (left) [right] invariant $X$ is (left) [ right] introverted if for every $x$ in $X$ and $\xi$ in $X^{*},\left(\xi_{l} x\right)\left[\xi_{r} x\right]$ are in $X\left(^{5}\right)$.

The following is a list of introverted spaces:

$m(\Sigma)$ is always introverted.

$C(\Sigma)$ is introverted if $\Sigma$ is a compact group.

$W(\Sigma)$ is introverted [6], where it is the subspace of $C(\Sigma)$ consisting of all weakly almost periodic functions.

$U_{l}(\Sigma)$ is left-introverted and $U_{r}(\Sigma)$ is right-introverted, where $U_{l}$ and $U_{r}$ are respectively the spaces of left and right uniformly continuous functions on $\Sigma$.

We note that if $\Sigma$ is compact or discrete then $C(\Sigma)$ is introverted; and $W$ which is a subspace of $C$ is introverted. This suggests that even if the space under consideration is not introverted, it may have (left) [ right] introverted subspaces and perhaps maximal ones among them. We show that this is the case and also give a characterization for the one-sided maximal introverted subspaces. First we state two lemmas whose proofs are completely straightforward.

LEMma 1. If $X$ is (l-) $\left[r_{-}\right]$invariant then so is $\bar{X}$, the norm closure.

LEMma 2. If $X$ is $(l-)\left[r_{-}\right]$introverted then so is $\bar{X}$.

From these two lemmas and the use of Zorn's Lemma it can be shown that any closed $(l-)\left[r_{-}\right]$invariant $X$ containing the constant functions has a maximal $\left(l_{-}\right)\left[r_{-}\right]$introverted subspace. However, we proceed in the following way to give a characterization of the one-sided maximal subspaces.

For a closed left invariant $X$, define $B_{l}=\left\{x \mid x \in X\right.$ and $\xi_{l} x \in X$ for all $\left.\xi \in X^{*}\right\}$, and similarly for a closed right invariant $X$ define $B_{r}=\{x \mid x \in X$ and $\xi_{r} x \in X$ for all $\left.\xi \in X^{*}\right\}$.

Theorem 1. (a) If $X$ is closed and left invariant then $B_{l}$ is the unique maximal left introverted subspace of $X$.

(b) If $X$ is closed and right invariant then $B_{r}$ is the unique maximal right introverted subspace of $X$.

The proof is divided into several lemmas.

Lemma 3. $B_{l}$ is a closed linear left invariant subspace of $X$.

Proof. Linearity is obvious. If $\left\{x_{n}\right\} \subset B_{l}$ and $\left\|x_{n}-x\right\| \rightarrow 0$ then for any $\xi \in X^{*}, \quad\left\|\xi_{l} x_{n}-\xi_{l} x\right\| \leqq\|\xi\|\left\|x_{n}-x\right\| \rightarrow 0$. By hypothesis $\xi_{l} x_{n} \in X$ for

( $\left.{ }^{5}\right) X$ is a subspace of $m(\Sigma)$ or $M B(\Sigma)$. Every element of $X^{*}$ can be considered as the restriction to $X$ of a suitable element of $m(\Sigma)^{*}$ or $M B(\Sigma)^{*}$. For convenience we will often do the following: when we have to consider an arbitrary element of $X^{*}$ we will instead consider an arbitrary element of $m(\Sigma)^{*}$ or $M B(\Sigma)^{*}$, which amounts to the same thing as long as we evaluate it at elements of $X$ only. 
all $n$ and $X$ is closed and so $\xi_{l} x \in X$ which means $x \in B_{l}$. Hence $B_{l}$ is closed. Finally, if $x \in B_{l}$ and $\sigma \in \Sigma$ then for any $\xi \in X^{*}, \xi_{l}\left(l_{\sigma} x\right)=l_{\sigma}\left(\xi(x)\right.$. So $l_{\sigma} x \in B_{l}$ which means $B_{l}$ is left invariant.

Lemma 4. If $x \in B_{l}$ and $\xi, \eta \in X^{*}$ then there exists a $\zeta \in X^{*}$ such that $\zeta_{l} x$ $=\eta_{l}\left(\xi_{l} x\right)$.

Proof. For any $x$ in $B_{l}$ define $\zeta^{\prime}(x)=\eta\left(\xi_{l} x\right)$. If $x_{1}, x_{2} \in B_{l}$ and $a$ and $b$ are real numbers then

$$
\begin{aligned}
\zeta^{\prime}\left(a x_{1}+b x_{2}\right) & =\eta\left(\xi_{l}\left(a x_{1}+b x_{2}\right)\right) \\
& =\eta\left(a \xi_{l} x_{1}+b \xi_{l} x_{2}\right)=a \eta\left(\xi_{l} x_{1}\right)+b \eta\left(\xi x_{2}\right)=a \zeta^{\prime}\left(x_{1}\right)+b \zeta^{\prime}\left(x_{2}\right)
\end{aligned}
$$

and also

$$
\begin{aligned}
\left\|\zeta^{\prime}\right\| & =\sup _{\|x\| \leqq 1 ; x \in B_{l}}\left|\zeta^{\prime}(x)\right|=\sup _{\|x\| \leqq 1} \mid \eta(\xi(x) \mid \\
& \leqq \sup _{\|x\| \leqq 1}\|\eta\|\|\xi x\| \leqq\|\eta\| \cdot\|\xi\|,
\end{aligned}
$$

which proves $\zeta^{\prime}$ is a bounded linear functional on $B_{l}$. Let $\zeta$ be any extension of $\zeta^{\prime}$ to all of $X$. Now if $x \in B_{l}$, then for any $\sigma \in \Sigma, \quad\left(\zeta_{l} x\right)(\sigma)=\zeta\left(l_{\sigma} x\right)$ $=\zeta^{\prime}\left(l_{\sigma} x\right)=\eta\left[\xi_{l}\left(l_{\sigma} x\right)\right]=\eta\left[l_{\sigma}\left(\xi_{l} x\right)\right]=\left[\eta_{l}\left(\xi_{l} x\right)\right](\sigma)$, that is, $\zeta_{l} x=\eta_{l}\left(\xi_{l} x\right)$, which is the required result.

Lemma 5. $B_{l}$ is the unique maximal left introverted subspace of $X$.

Proof. Let $x \in B_{l}$ and $\xi \in X^{*}$. By definition of $B_{l}, y=\xi_{l} x \in X$. To prove that $B_{l}$ is left introverted we need to show that $y \in B_{l}$. For this purpose, if $\eta$ is any element of $X^{*}$, then by Lemma 4 there exists a $\zeta \in X^{*}$ so that $\eta_{l} y=\zeta_{l} x$. But $\zeta_{l} x \in X$ and hence so does $\eta_{l} y$ which means $y=\xi_{l} x \in B_{l}$.

Now let, if possible, $N$ be a left introverted subspace such that $B_{l} \subsetneq N$ $\subseteq X$. Then there exists an $x \in N$ but $\notin B_{l}$. This means that there exists a $\xi \in X^{*}$ such that $\xi_{l} x \notin X$. Since $N \subseteq X$, it follows that $\xi_{l} x \notin N$ which makes it impossible for $N$ to be introverted, against our assumption. Hence $B_{l}$ is maximal. Also the above argument shows that any $x \notin B_{l}$ cannot be in any introverted subspace of $X$. This implies $B_{l}$ is the only maximal introverted subspace of $X$. This completes the proof of Lemma 5 and Part (a) of Theorem 1. Proof of (b) is similar.

The characterization for the maximal left introverted subspace given above is entirely in terms of $X$ and $X^{*}$ and as such it does not tell us what the subspace is. For example, if $X=C(\Sigma)$, then we know that $W$ $\subseteq U_{l} \subseteq B_{l}$. A legitimate question to ask would be if $B_{l}=U_{l}$ ? In the following theorem we give a partial answer to this question. In particular, we show that the maximal introverted subspace of the space of bounded continuous functions on the real line is the space of uniformly continuous functions. 
THEOREM 2. If $\Sigma$ is a group complete in an invariant metric and $B_{l}$ denotes the maximal left introverted subspace of $C(\Sigma)$ then $B_{l}=U_{l}$, the subspace consisting of left uniformly continous functions on $\Sigma$.

Proof. It is sufficient to prove $B_{l} \subseteq U_{l}$. We shall do so by showing that if $x \notin U_{l}$ then $x \notin B_{l}$. We start by introducing some notation. Let $d$ denote the metric. For $\sigma \in \Sigma$ and $t>0$, the set $S=S(\sigma ; t)=\{\tau \mid d(\sigma, \tau)<t\}$ is called the ball of radius $t$ and center $\sigma$. The set $\bar{S}=\{\tau \mid d(\sigma, \tau) \leqq t\}$ is called the closed ball and $S_{0}=S-\{\sigma\}$ is called the punctured ball.

If $x \notin U_{l}$ then there exist sequences $\left\{\sigma_{n}\right\}$ and $\left\{\tau_{n}\right\}$ such that (i) $d\left(\sigma_{n}, \tau_{n}\right)$ $\rightarrow 0$, (ii) $x\left(\sigma_{n}\right) \rightarrow A$ and (iii) $x\left(\tau_{n}\right) \rightarrow A^{\prime} \neq A$. We can suppose $A=1$ and $A^{\prime}=0$. Let $y_{n}=r_{\sigma_{n}} x$ and $\epsilon_{n}=\tau_{n} \sigma_{n}^{-1}$ and let $i$ denote the identity of $\Sigma$. $y_{n}(i) \rightarrow 1$ and $y_{n}\left(\epsilon_{n}\right) \rightarrow 0$. Now we must have either

$\left(\mathrm{a}_{1}\right)$ for each $\rho \in S_{0}(i ; 1)$ there exists an integer $N=N_{\rho}$ such that $y_{n}(\rho)$ $\geqq 1 / 2$ for all $n \geqq N$; or

$\left(b_{1}\right)$ there exists a subsequence $\left\{y_{n_{i}}\right\}$ of $\left\{y_{n}\right\}$ and a $\rho_{1} \in S_{0}(i ; 1)$ such that $y_{n_{i}}\left(\rho_{1}\right) \rightarrow p_{1} \leqq 1 / 2$.

If $\left(\mathrm{a}_{1}\right)$, let $E_{n}=\left\{\rho \mid y_{n}(\rho) \geqq 1 / 2, \rho \in S_{0}(i ; 1)\right]$ and $F_{n}=\bigcap_{k \geqq n} E_{k}$. Since any $\rho$ in $S_{0}$ will be in $E_{n}$ for all sufficiently large $n$, we have $\bigcup_{n} F_{n}=S_{0}(i ; 1)$. If $K$ is any closed ball in $S_{0}$ then $\bigcup_{n}\left(F_{n} \cap K\right)=K . F_{n} \cap K$ is closed for each $n$ and $K$ is complete metric and so is of second category in itself. Hence for some $N^{\prime}, F_{N^{\prime}} \cap K$ contains a ball $K^{\prime}=S(\alpha ; q)$. Since $\epsilon_{n} \rightarrow i$, we can choose $N^{\prime \prime}$ so that $\epsilon_{n} \in S(i ; q)$ for $n \geqq N^{\prime \prime}$. Thus for $n \geqq N=\max \left(N^{\prime}, N^{\prime \prime}\right)$ we have $\alpha \epsilon_{n} \in S(\alpha ; q)$ and hence $y_{n}\left(\alpha \epsilon_{n}\right) \geqq 1 / 2$ that is $\left(l_{\alpha} y_{n}\right)\left(\epsilon_{n}\right) \geqq 1 / 2$.

Now choose a sequence $\left\{\beta_{m}\right\} \subset \Sigma$ such that $\lim _{m} \beta_{m}=i$ and $0<d\left(\beta_{m}, i\right)$ $=t_{m}<1$. Let $S_{m}=S\left(\beta_{m} ; t_{m} / 2\right)$. By taking $\bar{S}_{m}$ for $K$ in the previous argument we get a sequence $\left\{\alpha_{m}\right\}$ such that: (iv) $\alpha_{m} \rightarrow i$ and (v) to each $m$ corresponds an integer $N_{m}$ such that $\left(l_{\alpha_{m}} y_{n}\right)\left(\epsilon_{n}\right) \geqq 1 / 2$ for all $n \geqq N_{m}$. Recall that $y_{n}\left(\epsilon_{n}\right)$ $=x\left(\tau_{n}\right) \rightarrow 0$. From the net (sequence) $\left\{\tau_{n}\right\}$ we can pick a subnet $\left\{\tau_{\sigma}\right\}$ such that this subnet converges to a point $\xi$ of the Cech compactification $\left({ }^{6}\right)$ of $\Sigma$. By definition $\xi \in C(\Sigma)^{*}$. Now for the function $\xi_{l} x$ we have:

$$
\left(\xi_{l} x\right)(i)=\xi\left(l_{i} x\right)=\xi(x)=\lim _{\delta} x\left(\tau_{\delta}\right)=\lim _{n} x\left(\tau_{n}\right)=0 ;
$$

and

$$
\begin{aligned}
\left(\xi_{l} x\right)\left(\alpha_{m}\right)=\xi\left(l_{\alpha_{m}} x\right) & =\lim _{n}\left(l_{\alpha_{m}} x\right)\left(\tau_{n}\right)=\lim _{n}\left(l_{\alpha_{m}} r_{\sigma_{n}} x\right)\left(\tau_{n} \sigma_{n}^{-1}\right) \\
& =\lim _{n}\left(l_{\alpha_{m}} y_{n}\right)\left(\epsilon_{n}\right) \geqq 1 / 2
\end{aligned}
$$

$\left({ }^{6}\right)$ Here we identify the elements $\tau$ of $\Sigma$ with the corresponding unit point masses (which lie in $\left.C(\Sigma)^{*}\right)$; the weak* closure of the set of all such point masses (in the weak* topology) can of course be identified with the Čech compactification of $\Sigma$. 
for all $m$. This is in virtue of (v) above. Since $\alpha_{m} \rightarrow i$, $\xi_{l} x$ is not continuous at $i$ and so $\notin C(\Sigma)$ and hence $x \notin B_{\text {l }}$.

Next let us consider the case $\left(b_{1}\right)$. In this case there exists a subsequence $\left\{y_{n_{i}}\right\}$ of $\left\{y_{n}\right\}$ and a $\rho_{1} \in S_{0}(i ; 1)$ such that $y_{n_{i}}\left(\rho_{1}\right) \rightarrow p_{1} \leqq 1 / 2$. Consider the punctured ball $S_{0}^{(2)}=S_{0}\left(i ; d\left(i, \rho_{1}\right) / 2\right)$ and again either: $\left(\mathrm{a}_{2}\right)$ for each $\rho$ $\in S_{0}^{(2)}$ corresponds an integer $N_{\rho}^{(2)}$ such that $y_{n_{i}}(\rho) \geqq 1 / 2$ for all $n \geqq N_{\rho}^{(2)}$; or $\left(b_{2}\right)$ there exists a subsequence $\left\{y_{n_{i_{j}}}\right\}$ of $\left\{y_{n_{i}}\right\}$ and a $\rho_{2} \in S_{0}^{(2)}$ such that $y_{n_{i}}\left(\rho_{2}\right) \rightarrow p_{2} \leqq 1 / 2$. If $\left(a_{2}\right)$ we proceed just as in $\left(a_{1}\right)$. If $\left(b_{2}\right)$ we consider the punctured ball $S_{0}^{(3)}=S_{0}\left(i ; d\left(i, \rho_{2}\right) / 2\right)$ and cases $\left(a_{3}\right)$ and $\left(b_{3}\right)$; and so on. This process will either stop at a case (a) or it will always be case (b) and we will have (vi) a sequence $\left\{\rho_{m}\right\}$ such that $\rho_{m} \rightarrow i$; (vii) a sequence of sequences $\{y\}$ where each one is a subsequence of the preceding one. By Cantor's diagonalisation process we get a sequence $\left\{y_{s}\right\}$ with the properties: (viii) $y_{s}(i)=x\left(\sigma_{s}\right) \rightarrow 1$; and (ix) $y_{s}\left(\rho_{m}\right)=x\left(\rho_{m} \sigma_{s}\right) \rightarrow p_{m} \leqq 1 / 2$ for each $m$. From the net $\left\{\sigma_{s}\right\}$ extract a subnet $\left\{\sigma_{\delta}\right\}$ converging to a point $\eta$ of the Cech compactification of $\Sigma$. By definition $\eta \in C(\Sigma)^{*}$. For the function $\eta_{l} x$ we have: $\left(\eta_{l} x\right)(i)=\eta(x)=\lim _{\delta} x\left(\sigma_{\delta}\right)=\lim _{s} x\left(\sigma_{s}\right)=1 ; \quad$ and $\quad\left(\eta_{l} x\right)\left(\rho_{m}\right)$ $=\eta\left(l_{\rho_{m}} x\right)=\lim _{s}\left(l_{\rho_{m}} x\right)\left(\sigma_{s}\right)=\lim _{s} x\left(\rho_{m} \sigma_{s}\right)=p_{m} \leqq 1 / 2$ for all $m$. This is by virtue of (ix) above. Since $\rho_{m} \rightarrow i, \eta_{l} x$ is not continuous at $i$ and so $\notin C(\Sigma)$ and hence $x \notin B_{l}$. Thus in any case if $x \notin U_{l}$ then $x \notin B_{l}$ and the proof is complete.

The remainder of this section is devoted to some of the properties and consequences of introversion. If $f: \Sigma \rightarrow \Sigma^{\prime}$ is a homomorphism then it induces a linear isometry $F$ from $m\left(\Sigma^{\prime}\right)$ into $m(\Sigma)$ defined by $\left(F x^{\prime}\right)(\sigma)=x^{\prime}(f \sigma)$. $F^{*}$, the conjugate operator, has norm one. See Day [5]. Since our interest is not in $m$ but in spaces like $C$ and $M B$ we specialize on $F$ and get

Theorem 3. If $X^{\prime}$ is a closed linear introverted subspace of $C\left(\Sigma^{\prime}\right)\left[M B\left(\Sigma^{\prime}\right)\right]$ and $f$ is a continuous [measurable] homomorphism of $\Sigma$ into $\Sigma^{\prime}$ then $F\left(X^{\prime}\right)$ is a closed linear introverted subspace of $C(\Sigma)[M B(\Sigma)]$.

Proof. For simplicity let $X=F\left(X^{\prime}\right)$. It is easy to see that $X$ is closed and linear. To show that $X$ is introverted, we have to prove that for any $x \in X$ and $\xi \in C(\Sigma)^{*}\left[M B(\Sigma)^{*}\right], \xi_{1} x$ and $\xi_{\mu} x \in X$. Since $x \in X=F\left(X^{\prime}\right)$, there exists an $x^{\prime} \in X^{\prime}$ such that $x=F x^{\prime}$. For any $\sigma$ and $\sigma_{1} \in \Sigma,\left(l_{\sigma} x\right)\left(\sigma_{1}\right)$ $=\left(F x^{\prime}\right)\left(\sigma \sigma_{1}\right)=x^{\prime}\left(f\left(\sigma \sigma_{1}\right)\right)=x^{\prime}\left(\sigma^{\prime} \sigma_{1}^{\prime}\right)=\left(l_{\sigma^{\prime}} x^{\prime}\right)\left(\sigma_{1}^{\prime}\right)=\left(F l_{\sigma^{\prime}} x^{\prime}\right)\left(\sigma_{1}\right)$, which means $l_{\sigma} F x^{\prime}=F l_{\sigma^{\prime}} x^{\prime}$. Hence for every $\sigma$ in $\Sigma,(\xi x)(\sigma)=\xi\left(l_{\sigma} x\right)=\xi\left(l_{\sigma} F x^{\prime}\right)=\xi\left(F l_{\sigma^{\prime}} x^{\prime}\right)$ $=\left(F^{*} \xi\right)\left(l_{\sigma^{\prime}} x^{\prime}\right)=\left(\xi_{l}^{\prime} x^{\prime}\right)\left(\sigma^{\prime}\right)=\left(F \xi_{l}^{\prime} x^{\prime}\right)(\sigma)$. That is, $\xi_{l} x=F \xi_{l}^{\prime} x^{\prime}$. Since $X^{\prime}$ is introverted, $\xi_{l}^{\prime} x^{\prime} \in X^{\prime}$ and hence $\xi_{l} x \in X$. Similarly we show $\xi_{r} x \in X$.

The above theorem (roughly) says that introversion is preserved under homomorphism. A similar theorem can be proved for the operator II (see Day [5]) which is a special case of $F$. 
One of the most important consequences of introversion is that if $X$ is introverted then a multiplication can be defined on $X^{*}$ to make it a Banach algebra. This follows the method given by Arens [1] and modified by Day [5] and used by Rosen [8]. It is in three steps: For each $x \in X$ and $\sigma \in \Sigma$ let $x \odot \sigma=l_{\sigma} x$; for $x \in X$ and $\eta \in X^{*}$, let $(\eta \odot x)(\sigma)=\eta(x \odot \sigma)=\eta\left(l_{\sigma} x\right)$ $=\left(\eta_{l} x\right)(\sigma)$, that is $\eta \odot x=\eta_{l} x$; for $\xi, \eta \in X^{*}$ define $(\xi \odot \eta)=(x) \xi(\eta \odot x)$ for all $x \in X$. Note that $x \odot \sigma$ belongs to $X$ because $X$ is invariant, and $\eta \odot x$ is in $X$ because $X$ is introverted. It is easy to check that $\xi \odot \eta$ belongs to $X^{*}$. We omit the proof that $X^{*}$ is a Banach algebra under $\odot$ because it is the same as that of Lemma 6.1 of Day [5]. However, notice that in the above definition only left introversion is used. We could, obviously, define a similar multiplication with the first step $x \diamond \sigma=r_{\sigma} x$ and follow it up by $\eta \diamond x=\eta_{r} x$ and $(\xi \diamond \eta)(x)=\xi\left(\eta_{r} x\right)$. Clearly $\diamond$ is as good as $\odot$. Rosen [8] remarks that, in general, these two do not coincide. In the following theorem we present a necessary and sufficient condition for the two multiplications to agree on $B^{*}$, where $B$ is the maximal introverted subspace of $C(\Sigma)$.

TheOREM 4. $\odot$ and $\diamond$ coincide on $B^{*}$ if and only if $\Sigma$ is abelian.

Proof. If $\Sigma$ is abelian $l_{\sigma} x$ and $r_{\sigma} x$ are the same and the definitions coincide all the way through. On the other hand, let $\xi \odot \eta=\xi \diamond \eta$ for all $\xi, \eta \in B^{*}$. Let $\sigma_{1}$ and $\sigma_{2}$ be two (distinct) points of $\Sigma$ and define the functionals $\xi, \eta$ as the evaluations at $\sigma_{1}$ and $\sigma_{2}$ respectively, that is for any $x \in C(\Sigma), \xi(x)$ $=x\left(\sigma_{1}\right)$ and $\eta(x)=x\left(\sigma_{2}\right)$. Now we must have for any $x \in B,(\xi \odot \eta)(x)$ $=(\xi \diamond \eta)(x)$, that is, $\xi\left(\eta_{l} x\right)=\xi\left(\eta_{r} x\right)$ which means $\left(\eta_{l} x\right)\left(\sigma_{1}\right)=\left(\eta_{r} x\right)\left(\sigma_{1}\right)$. This last equality can be written as $\eta\left(l_{\sigma_{1}} x\right)=\eta\left(r_{\sigma_{1}} x\right)$, that is, $\left(l_{\sigma_{1}} x\right)\left(\sigma_{2}\right)$ $=\left(r_{\sigma_{1}} x\right)\left(\sigma_{2}\right)$ which means $x\left(\sigma_{1} \sigma_{2}\right)=x\left(\sigma_{2} \sigma_{1}\right)$. As we know $B \supseteq W$ and $W$ contains $C_{0}$, the subspace of $C(\Sigma)$ consisting of all continous functions vanishing at the Alexandrov point. For any Hausdorff topological group, $C_{0}$ separates points and hence it follows from the last equation that $\sigma_{1} \sigma_{2}$ $=\sigma_{2} \sigma_{1}$. This being true for any pair of points, $\Sigma$ is abelian. (Note: Obviously we could prove the theorem for any introverted $X$ provided it has enough functions to separate points.)

We conclude this section with a result which throws more light on a remark made in the previous one. Let, as in the above theorem, $B$ denote the maximal introverted subspace of $C(\Sigma)$ where $\Sigma$ is a Hausdorff locally compact topological group. Consider the map $R: \Sigma \rightarrow \mathscr{L}(B)$ defined by $R(\sigma)=r_{\sigma}$. We know that $R$ is an algebraic homomorphism. Now we prove

Theorem 5. $R$ is an isomorphism of the topological group $\Sigma$ into the multiplicative (topological) semigroup $\mathscr{L}(B)$ equipped with the weak operator topology.

Proof. Recall that $B$ separates points. So, given $\sigma \neq \sigma^{\prime}$, there exists an $x \in B$ such that $x(\sigma) \neq x\left(\sigma^{\prime}\right)$, that is, $\left(r_{\sigma} x\right)(i) \neq\left(r_{\sigma^{\prime}} x\right)(i)$ where $i$ is the 
identity of $\Sigma$. Hence $r_{\sigma} \neq r_{\sigma^{\prime}}$ and the map $R$ is univalent. Let $R(\Sigma)$ denote the image of $\Sigma$ in $\mathscr{L}(B)$, under the map $R$. That is

$$
R(\Sigma)=\left\{r_{\sigma} \mid \sigma \in \Sigma, r_{\sigma} \in \mathscr{L}(B)\right\} .
$$

Topologise $R(\Sigma)$ by relativising the weak operator topology of $\mathscr{L}(B)$. To complete the proof we have to show that $\Sigma$ and $R(\Sigma)$ are homeomorphic. $B$ introverted implies $R$ is continuous. We will show $R$ open. For this purpose, let $U$ be an open set of $\Sigma$ and $\sigma^{\prime} \in U$. Choose any neighborhood $V$ of the identity $i$ such that $V \sigma^{\prime} \subset U$. Since the group is locally compact we can select a neighborhood $W$ of $i$ such that $\bar{W}$ is compact and $W^{2} \subset V$. Let $S$ be the complement of $W \sigma^{\prime} . S$ is closed and $\sigma^{\prime} \notin S$. By the complete regularity of $\Sigma$, there exists an $x \in C(\Sigma)$ such that $x\left(\sigma^{\prime}\right)=1$ and $x(S)=0$ and $0 \leqq x \leqq 1$. Obviously, $x$ vanishes outside $\overline{W \sigma^{\prime}}$, a compact set, and hence $x \in C_{0}(\Sigma) \subseteq B$. Define $\xi$ as $\xi(y)=y(i)$ for all $y \in C(\Sigma)$. Now consider the following neighborhood of $r_{\sigma^{\prime}}$ :

$$
\begin{aligned}
N_{\sigma^{\prime}} & =N\left(r_{\sigma^{\prime}} ; x ; \xi ; 1 / 2\right) \\
& =\left\{r_{\sigma}|| \xi\left(r_{\sigma} x\right)-\xi\left(r_{\sigma^{\prime}} x\right)|=|\left(r_{\sigma} x\right)(i)-\left(r_{\sigma^{\prime}} x\right)(i) \mid<1 / 2\right\} \\
& =\left\{r_{\sigma}|| x(\sigma)-x\left(\sigma^{\prime}\right) \mid=1-x(\sigma)<1 / 2\right\} .
\end{aligned}
$$

Since $x$ is zero outside $W \sigma^{\prime}$ it follows that $N_{\sigma^{\prime}} \subset R\left(W \sigma^{\prime}\right) \subset R(U)$. This proves that $R$ is open and the proof is complete.

3. Amenability. We start with two lemmas dealing with products of means in the $\odot$ multiplication. The means considered here are over an introverted $X$ and so the $\odot$ multiplication is defined on $X^{*}$.

LemMa 6. If $\mu$ and $\nu$ are means then $\mu \odot \nu$ is a mean.

Proof. Direct from definition.

Lemma 7. $\mu \odot \nu$ is left invariant if $\mu$ is left invariant and is right invariant if $\nu$ is right invariant.

Proof. For any $\sigma \in \Sigma, x \in X$ and $\nu \in X^{*}$ we have $\nu \odot l_{\sigma} x=\nu_{l}\left(l_{\sigma} x\right)=l_{\sigma}\left(\nu_{l} x\right)$ $=l_{\sigma}(\nu \odot x)$. Hence if $\mu$ is left invariant then

$$
(\mu \odot \nu)\left(l_{\sigma} x\right)=\mu\left(\nu \odot l_{\sigma} x\right)=\mu\left(l_{\sigma}(\nu \odot x)\right)=\mu(\nu \odot x)=(\mu \odot \nu)(x),
$$

which proves that $\mu \odot \nu$ is left invariant.

On the other hand if $\nu$ is right invariant, then for any $\sigma^{\prime} \in \Sigma,\left(\nu \odot r_{\sigma} x\right)\left(\sigma^{\prime}\right)$ $=\nu\left(l_{\sigma^{\prime}} r_{\sigma} x\right)=\nu\left(r_{\sigma} l_{\sigma^{\prime}} x\right)=\nu\left(l_{\sigma^{\prime}} x\right)=(\nu \odot x)\left(\sigma^{\prime}\right)$, that is, $\nu \odot r_{\sigma} x=\nu \odot x$ and $(\mu \odot \nu)\left(r_{\sigma} x\right)=\mu\left(\nu \odot r_{\sigma} x\right)=\mu(\nu \odot x)=(\mu \odot \nu)(x)$, which proves that $\mu \odot \nu$ is right invariant. From these two lemmas we get

Theorem 6. If an introverted $X$ is both right and left amenable then it is amenable. 
Proof. If $\lambda$ and $\rho$ are respectively left and right invariant means on $X$ then $\lambda \odot \rho$ is a two-sided invariant mean.

Several results similar to those in Day [5] are valid for an introverted $X$. Thus for example Theorem 1 and Corollaries 4, 5, and 6 of $\$ 6$ in [5] are valid if $m(\Sigma)$ is replaced by any introverted $X$. Theorem 6 above is similar to Proposition (A) of $\$ 4$ of the same paper. Proposition (B) can be proved in the present context also. Analogous to $(\mathrm{H})$ and $(\mathrm{I})$, it can be shown that if $\Sigma$ is abelian or compact then both $C(\Sigma)$ and $M B(\Sigma)$ are amenable.

\section{REFERENCES}

1. R. Arens, The adjoint of a bilinear operator, Proc. Amer. Math. Soc. 2 (1951), 839-848.

2. S. Banäch, Théorie des opérations linéaires, Monogr. Mat., Tom 1, Warsaw, 1932.

3. N. Bourbaki, Eléments de mathématique. XVII, Livre V, Espaces vectoriels topologiques, Chapitres III-V, Actualités Sci. Indust. No. 1229, Hermann, Paris, 1955.

4. M. M. Day, Means for bounded functions and ergodicity of the bounded representations of semigroups, Trans. Amer. Math. Soc. 69 (1956), 276-291.

5. __ Amenable semigroups, Illinois J. Math. 1 (1957), 509-544.

6. I. Glicksberg and K. deLeeuw, Applications of almost periodic compactifications, Technical Note No. 9 (AFOSR TN 59-1174), Stanford Univ., Stanford, Calif., 1959.

7. J. von Neumann, Zur allgemeine Theorie des Masses, Fund. Math. 13 (1929), 167-190.

8. W. G. Rosen, On invariant means over topological semigroups, Ph.d Thesis, Univ. of Illinois, Urbana, Illinois, 1954.

UNIVERSITY OF ILLINOIS, URBANA, ILLINOIS

UNIVERSITY OF MiChIGAN, ANn Arbor, Michigan 